\title{
Radioligands for Tropomyosin Receptor Kinase (Trk) Positron Emission Tomography Imaging
}

\author{
Ralf Schirrmacher ${ }^{1, *} \mathbb{( D}$, Justin J. Bailey ${ }^{1}$, Andrew V. Mossine ${ }^{2}$, Peter J. H. Scott ${ }^{2,3}$ (D),
} Lena Kaiser ${ }^{4}{ }^{(0)}$, Peter Bartenstein ${ }^{4}$, Simon Lindner ${ }^{4}$, David R. Kaplan ${ }^{5}$, Alexey Kostikov ${ }^{6}$, Gert Fricker ${ }^{7}$, Anne Mahringer ${ }^{7}$, Pedro Rosa-Neto ${ }^{8}$, Esther Schirrmacher ${ }^{1}$, Carmen Wängler ${ }^{9}$, Björn Wängler ${ }^{10}$, Alexander Thiel ${ }^{6,11}$, Jean-Paul Soucy ${ }^{6}$ and Vadim Bernard-Gauthier ${ }^{12,13, *}$

1 Department of Oncology, Division of Oncological Imaging, University of Alberta, Edmonton, Alberta T6G 2R3, Canada; jjbailey@ualberta.ca (J.J.B.); eschirrm@ualberta.ca (E.S.)

2 Division of Nuclear Medicine, Department of Radiology, The University of Michigan Medical School, Ann Arbor, MI, 48109, USA; amossine@med.umich.edu (A.V.M.); pjhscott@med.umich.edu (P.J.H.S.)

3 The Interdepartmental Program in Medicinal Chemistry, University of Michigan, Ann Arbor, MI 48109, USA

4 Department of Nuclear Medicine, Ludwig-Maximilians-University of Munich, Marchioninistrasse 15, Munich 81377, Germany; Lena.Kaiser@med.uni-muenchen.de (L.K.);

Peter.Bartenstein@med.uni-muenchen.de (P.B.); Simon.Lindner@med.uni-muenchen.de (S.L.)

5 Program in Neurosciences and Mental Health, Hospital for Sick Children and Department of Molecular Genetics, University of Toronto, Toronto, ON, Canada M5G 0A4; dkaplan@sickkids.ca

6 McConnell Brain Imaging Centre, Montreal Neurological Institute, McGill University, 3801 University Street, Montreal, QC H3A 2B4, Canada; alexey.kostikov@mcgill.ca (A.K.); alexander.thiel@mcgill.ca (A.T.); jean-paul.soucy@mcgill.ca (J.-P.S.)

7 Institute of Pharmacy and Molecular Biotechnology, University of Heidelberg, Heidelberg 69120, Germany; gert.fricker@uni-hd.de (G.F.); mahringer@uni-hd.de (A.M.)

8 Translational Neuroimaging Laboratory, McGill Centre for Studies in Aging, Douglas Mental Health University Institute, Montreal, QC H4H 1R3, Canada; pedro.rosa.neto@gmail.com

9 Biomedical Chemistry, Department of Clinical Radiology and Nuclear Medicine, Medical Faculty Mannheim of Heidelberg University, 68167 Mannheim, Germany; Carmen.Waengler@medma.uni-heidelberg.de

10 Molecular Imaging and Radiochemistry, Department of Clinical Radiology and Nuclear Medicine, Medical Faculty Mannheim of Heidelberg University, Theodor-Kutzer-Ufer 1-3, Mannheim 68167, Germany; Bjoern.Waengler@medma.uni-heidelberg.de

11 Jewish General Hospital, Lady Davis Institute, Montreal, QC HT3 1E2, Canada

12 Azrieli Centre for Neuro-Radiochemistry, Research Imaging Centre, Centre for Addiction and Mental Health, Toronto, ON M5T 1L8, Canada

13 Department of Psychiatry, University of Toronto, Toronto, ON M5T 1R8, Canada

* Correspondence: schirrma@ualberta.ca (R.S.); Vadim.bernard-gauthier@camhpet.ca (V.B.-G.); Tel.: +1-780-235-8578 (R.S.)

Received: 27 November 2018; Accepted: 17 December 2018; Published: 3 January 2019

\begin{abstract}
The tropomyosin receptor kinases family (TrkA, TrkB, and TrkC) supports neuronal growth, survival, and differentiation during development, adult life, and aging. TrkA/B/C downregulation is a prominent hallmark of various neurological disorders including Alzheimer's disease (AD). Abnormally expressed or overexpressed full-length or oncogenic fusion TrkA/B/C proteins were shown to drive tumorigenesis in a variety of neurogenic and non-neurogenic human cancers and are currently the focus of intensive clinical research. Neurologic and oncologic studies of the spatiotemporal alterations in TrkA/B/C expression and density and the determination of target engagement of emerging antineoplastic clinical inhibitors in normal and diseased tissue are crucially needed but have remained largely unexplored due to the lack of suitable non-invasive probes. Here, we review the recent development of carbon-11- and fluorine-18-labeled positron emission tomography (PET) radioligands based on specifically designed small molecule kinase catalytic domain-binding inhibitors of TrkA/B/C. Basic developments in medicinal chemistry, radiolabeling and translational PET imaging in multiple species including humans are highlighted.
\end{abstract}


Keywords: tropomyosin receptor kinase; positron emission tomography; neurodegeneration; oncogenic fusions

\section{Introduction}

Tropomyosin receptor kinases (TrkA, TrkB, and TrkC) are transmembrane glycoproteins encoded by genes NTRK1-3, respectively. These kinases encompass extracellular domains (ECD) interacting specifically with endogenous neurotrophins as well as highly homologous intracellular tyrosine kinase domains (Figure 1A). Nerve growth factor (NGF) binds to TrkA, brain-derived neurotrophic factor (BDNF), neurothrophin-3 (NT-3), and neurotrophin-4 (NT-4) to TrkB, and NT-3 to TrkC [1-3]. Full length TrkB is a $140-\mathrm{kD}$ transmembrane spanning protein, with an extracellular ligand-binding domain containing two cysteine clusters, leucine-rich repeats, and two immunogloblulin-like domains [4]. The intracellular domain encodes a tyrosine kinase domain that when activated transphosphorylates monomers of the TrkB dimer. When transphosphorylated, the Trks engage and phosphorylate their major substrates Shc, Phospholipase C $\gamma 1$ (PLC- $\gamma$ 1), and Fibroblast Growth Factor Receptor Substrate 2 (FRS2/SNT1) [5]. The TrkB locus also encodes four variants generated by alternative splicing, of which the most abundant is the 90-kD truncated TrkB.t1 isoform that lacks the kinase domain [6]. Trk signaling occurs primarily through Ras/Mitogen-Activated Protein Kinase 1 (MAPK1), Phosphoinositide-3-Kinase (PI3-K)/Akt and PLC- $\gamma 1[4,7-9]$ and plays central roles in mediating neuronal survival and differentiation in the embryonic, postnatal, and mature peripheral (PNS) and central nervous system (CNS) [2,3]. Within the CNS, reduced expression, as well as abnormal and impaired signaling of Trk receptors, are associated with a plethora of neuropathologies, including ischemic brain injury, schizophrenia, Rett syndrome, depression, Parkinson's disease (PD), and Alzheimer's disease (AD) [10-16]. In AD, evidence from ex vivo experiments accumulated over the last two decades demonstrates reductions in full length catalytic TrkB/C receptor densities as well as a decline in TrkB/C neurotrophin signaling [17]. Direct evidence for the involvement of alterations in BDNF/TrkB signalling in $\mathrm{AD}$ is supported by studies indicating that TrkB levels are profoundly decreased in the hippocampus, frontal and temporal cortex of patients with Alzheimer's [18], although apparently not in the parietal cortex [19]. Furthermore, progressive loss of TrkA, $\mathrm{B}$, and $\mathrm{C}$ in basal forebrain cholinergic nuclei is well correlated with the clinical progression of $\mathrm{AD}$ [20]. Treatment with agonists of the BDNF/TrkB system of transgenic mouse models of AD increases dendritic spines in the hippocampus and cortex, inhibits neuronal apoptosis and neurodegeneration, and improves spatial memory performance [21-25]. These findings support the use of agents that activate BDNF/TrkB for treating AD [26]. Yet, the in vivo relevance or the spatiotemporal evolution of such changes, as well as the relationships which may exist with known neuropathological hallmarks of neurodegeneration such as plaque deposition and neurofibrillary tangle (NFTs) aggregation in AD-both of which can be visualized using diagnostic positron emission tomography (PET) - are unresolved questions. The potential importance of perturbations of Trk expression, activity and signalling in neurodegeneration hence raises the question of whether these molecular targets can in turn be imaged in vivo and non-invasively using PET. Another potential application for Trk-targeted radioligands stems from the renewed and rapidly growing interest in anti-Trk therapy for cancer. Indeed, important advances in recent years have been made in the treatment of patients with NTRK fusion-positive cancers in basket trials using pan-Trk inhibitors [27]. In parallel, remarkable progress has also been achieved in the development of selective pan-Trk and TrkA subtype-selective tyrosine kinase inhibitors (TKIs). NTRK fusions are found at low frequency in a number of common cancers and at a relatively high frequency in rare neoplasms-amounting to about 1500-5000 patients with NTRK fusions-positive diseases per year in the United States. Current clinical trials assessing NTRK fusion-positive patients inherently rely on tumour biopsy (which may not be always achievable) followed by next generation sequencing or fluorescence in situ hybridization for fusion detection. The use of Trk-targeted PET imaging in early clinical stages to assess receptor occupancy, dosing regimen, and NTRK fusion-positive status, or to monitor treatment response in place of sequential 
tumour biopsy may be both achievable and desirable—as previously done with other molecular targeted TKI therapies [28].
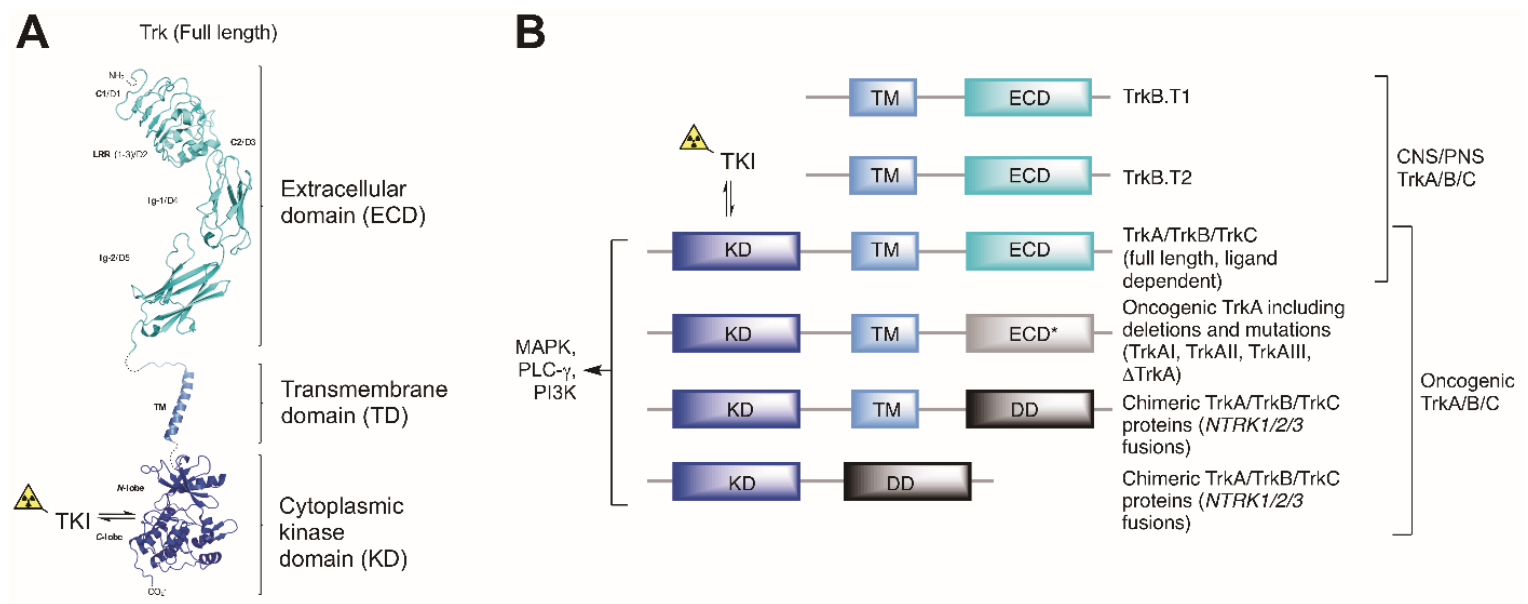

Figure 1. Detailed and representative domains of normally expressed and aberrantly expressed oncogenic tropomyosin receptor kinase (Trk) proteins from NTRK fusions (TKI: tyrosine kinase inhibitor). (A) Structure overview of the representative full TrkA receptor (D1-D5: domain 1-5; C1/3: cysteine cluster 1/3; LRR: leucine-rich repeat; Ig-1/2: immunoglobulin domain 1/2, TM: transmembrane domain). (B) Schematic representation of diverse Trk proteins and domains, including Trk splice variants and Trk fusion proteins. Dimerization and trans-autophosphorylation of Trk kinase domains leads to the activation of the downstream signaling pathways, including MAPK1, PI3-K/Akt and PLC- $\gamma 1$ (DD: dimerization domain).

Until very recently, suitable imaging lead compounds or quantifiable non-invasive techniques to measure spatiotemporal fluctuations of TrkA/B/C levels have been unavailable. To address this, we undertook in 2014 the task of identifying structural determinants which would enable TrkA/B/C PET imaging. To this end, we developed structurally diverse Trk radiotracers and inhibitor libraries with various levels of potency and kinome selectivity, both from type I and type II inhibitor classes, and exploited diverse radiochemical approaches using carbon- 11 and fluorine-18. While our primary objective has been non-oncological neuroimaging in the context of neurodegeneration and most results gathered thus far aimed at meeting this objective, we recognize that with the recent clinical oncological breakthrough in Trk inhibitor therapy comes a clear need for reliable and non-invasive assessment of Trk status in cancer therapy trials. In this short review, we describe the rational design and development of first-in-class Trk-targeted TKI PET radiotracers and delineate imaging validation obtained with these molecular probes to date.

\section{The Development of Trk Radioligands for PET Imaging}

\subsection{Binding Site Considerations}

PET radionuclides decay by emission of a positron, which in turn annihilates with a nearby electron, generating two gamma rays of $511 \mathrm{keV}$ (conversion of the positron's and electron's mass into energy). These two gamma rays, emitted in the opposite directions, can then be detected by an outside PET camera, revealing the position of the annihilation events with sufficient spatial resolution (from submillimeter to millimeter in preclinical and clinical settings respectively). Under the assumption of a sufficient tissue target receptor concentration (Bmax), an ideal radiotracer, in this context, needs to meet certain criteria which include: (1) high radioligand concentration in the tissue of interest, (2) radiotracer equilibrium conditions are reached, (3) lack of interfering radiometabolites and (4) high on-target selectivity. Beyond requiring careful studies of possible radiometabolites, this also highlights the importance of targeting suitable domains of a molecular target. This is especially relevant in the case of Trk where 
various isoforms, splice variants or fusions proteins may be co-expressed within a single tissue in different clinical contexts. For example, in addition to the full length TrkA/B/C, a number of truncated isoforms lacking the intracellular kinase domain have been characterized for TrkB (Figure 1B). Within the brain, the protein expression levels of the truncated isoforms of TrkB present (TrkB.T1 and TrkB.T2) far exceed that of full length TrkB [29]. A radiotracer binding the TrkB extracellular domain (ECD) would consequently map the sum of all TrkB receptors in the brain including the truncated isoforms which as yet have no known reported association with neurodegeneration or as oncogenic drivers. A PET signal originating from such a radiotracer would fail to differentiate catalytically-incompetent truncated from full-length kinase domain-containing receptors and would be unable to engage targets which lack cognate ectodomains such as the many NTRK fusion proteins which are of prime clinical interest (Figure 1B). More pragmatically, another reason to avoid targeting ECDs of Trk for imaging stems from the lack of suitable compounds. Indeed, while the study of nonprotein Trk ECD-binding compounds largely predates the advent of Trk-targeted TKIs, very limited progress has been achieved due to the inherently challenging druggability of the ectodomains compared to the kinase domains of TrkA/B/C and the poor drug-like properties of such compounds. These limitations have been exemplified by our imaging study of radiolabeled 7,8-dihydroxyflavone (7,8-DHF) [30]. This work was based on the initial report by Jang et al. suggesting that 7,8-DHF binds with high affinity to the ECD of TrkB and exhibits agonistic activity in neurons [31]. From this initial work, other more active flavones were described including fluorinated analogs which caught our attention as potential starting points for ${ }^{18} \mathrm{~F}-\mathrm{PET}$ tracer development [32,33]. Yet, using synthesized or commercially available described flavones we were unable to reproduce any basic biochemical effects in cells (unpublished data). In line with our findings, Boltaev et al. more recently unambiguously demonstrated the lack of direct interaction or TrkB-driven effect of such compounds [34]. In addition, we observed poor in vivo profiles in rats when investigating the radiolabeled isotopologs of these compounds for imaging (including 2-(4-[ $\left[{ }^{18} \mathrm{~F}\right]$ fluorophenyl)-7,8-dihydroxy-4H-chromen-4-one). Preclinical imaging revealed low-radioactivity overall brain uptake with a maximum standard uptake value (SUV) of 0.64 (whole brain) followed by fast elimination from the brain. These radioligands also were rapidly eliminated from the plasma compartment by hepatic metabolism with an estimated plasma half-life under 4 min suggesting a fast and extensive phase II metabolism and suggesting overall poor druglike properties, as it is known for hydroxyflavones more generally [35,36]. Interestingly, in vitro autoradiography with rat brains revealed apparent specific and BDNF-competitive binding reminiscent of ${ }^{125} \mathrm{I}$-BDNF binding topology. Yet, we surmise that this binding/blocking effect may have been a consequence of covalent reactivity to nucleophilic cysteines or other non-selective thiol-based reactivity as previously described for pan assay interference flavonoids [37]. Compared to putative ECD binding compounds, inhibitors targeting the ATP binding site of the cytoplasmic Trk kinase domains have been well characterized both functionally and structurally and have been reported alongside detailed crystallographic data enabling derivatization [38]. Targeting the kinase domains however comes with the challenge of selectivity, firstly within the kinome and secondly from within the Trk kinase family itself (TrkA, TrkB and TrkC) - TrkA/B/C display 72-78\% and 95-100\% sequence identity in their kinase domain and ATP binding sites, respectively. We have strived to detail kinome selectivity using comprehensive kinase screening whenever possible. With the exception of a few compounds, all radiolabeled inhibitors display pan-Trk activities. Yet, owing to the high expression levels of TrkB/C in the CNS compared to TrkA [39-43], and given that the PET signal is driven by target density and ligand affinity, it is to be expected that CNS PET using pan-Trk radioligands will nevertheless detect nearly exclusively TrkB/C-based signal. Another important note pertains to the fact that Trk kinases can adopt both DFG-in (active, targeted by type-I inhibitors) and DFG-out (inactive, targeted by type-II inhibitors) conformations (Figure 2). Conformational changes upon DFG triad rearrangement allow for the access of a deep pocket which can be occupied favorably by lipophilic moieties (Figure 2B,C). Type II Trk inhibitors targeting DFG-out conformation thus also present longer residence times compared to type-I inhibitors. To study potential benefits from each binding modes, we have developed both type-I and II radioligands. 
The following paragraphs give an account on our Trk radiotracer development program in chronological order progressing towards first in human PET imaging from preclinical research.

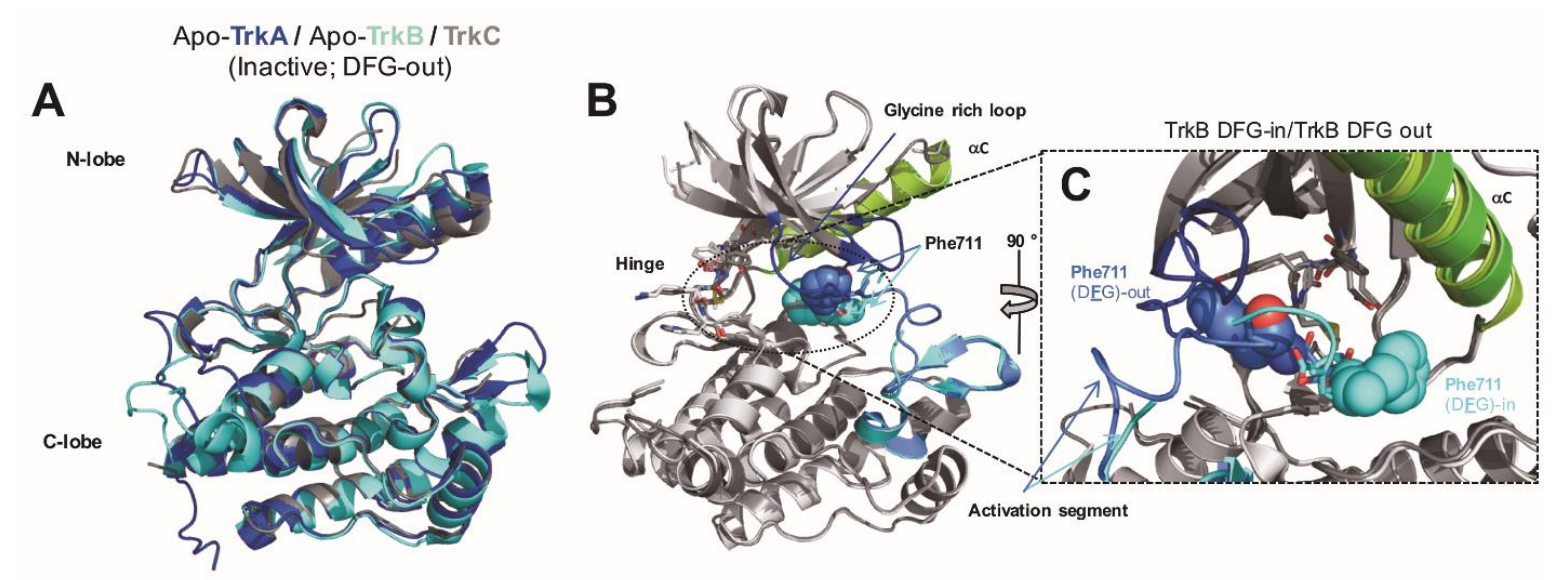

Figure 2. Trk kinase domain. (A) Overlap of TrkA, TrkB, and TrkC kinase domain (inactive conformations, PDB ID: 4F0I, 4ASZ, 3V5Q). (B,C) Views of the conformational differences between "Asp-Phe-Gly" DFG-in and DFG-out TrkB. The Phe residues of the DFG triad are shown in spheres (PDB ID: 4AT3, 4AT5).

\subsubsection{The 4-Aza-2-oxindole Radioligands}

PET tracers for neuroimaging have significantly higher chances of achieving high specific binding in brain when displaying certain physicochemical properties. Parameters such as rapid Blood Brain Barrier (BBB) permeation and neuron cell penetration as well as low non-specific binding are mostly determined by the compound's lipophilicity (represented as $\operatorname{cog} \mathrm{D}_{7.4}$ value, assuming passive diffusion through the cell membrane), reduced susceptibility to active efflux, molecular weight $(<350 \mathrm{Da})$, favorable neuroreceptor binding potential $\left(\mathrm{BP}=\mathrm{B}_{\max } / \mathrm{K}_{\mathrm{d}}>10\right)$, topological surface area (TSPA) of 30-80 $\AA^{2}$, and hydrogen bond donor ability (HBD) of $>1$. These general factors as well as specific properties tend to characterize successful neuroimaging PET tracers [44-47]. With these parameters in mind, we first opted for an inhibitor belonging to the family of 3-arylideneindolin-2-one Trk inhibitors. The 4-aza-2-oxindole inhibitor GW441756 [48] was screened as a compound favorably fulfilling the above mentioned requirements of a potentially successful candidate for brain imaging and was consequently labeled with carbon-11 to provide the radioactive isotopolog for autoradiography and PET imaging (half maximal inhibitory concentration $\left(\mathrm{IC}_{50}\right)$; $\operatorname{TrkA}=29.6 \mathrm{nM}$, TrkB $=6.7 \mathrm{nM}$, and TrkC $=4.6 \mathrm{nM}$, Figure 3) [49]. Even though the radiolabeling was straightforward and high yielding, the targeted $Z$ isomer, which based on molecular modeling was found to be the active isomer, could not be obtained in pure form after purification of the crude radiolabeling solution. An $E / Z$ 1:1 mixture was isolated under all conducted labeling conditions as a result of rapid in situ isomerisation either during the radiosynthesis or during the HPLC purification procedure, reducing the amount of high affinity Trk tracer Z-[ $\left.{ }^{11} \mathrm{C}\right] \mathrm{GW} 441756$ by $50 \%$. The inevitable contamination of the $E$-isomer with Z- $\left[{ }^{11} \mathrm{C}\right] \mathrm{GW} 441756$ significantly compromised the merit of this tracer as a potential Trk PET imaging agent, not only from an imaging quality perspective ( $50 \%$ of the PET signal will be the result of the non-specific binding of the Z-isomer) but also from a clinical translational point of view. Autoradiography of the mixed isomers on coronal rat brain sections revealed a displaceable binding in accordance with the ubiquitous distribution of all TrkB/C subtypes. The in vivo evaluation of $\left[{ }^{11} \mathrm{C}\right]$ GW441756 was performed in Sprague-Dawley rats to evaluate BBB penetration and in vivo biodistribution. In baseline scans the tracer rapidly entered the brain peaking at a SUV of 2 after $30 \mathrm{~s}$, after which the compound was rapidly eliminated from the brain over $30 \mathrm{~min}$. Tracer distribution was uniform in accordance with ubiquitous TrkB/C expression in the rodent brain. A blocking study using unlabeled GW441756 pre-treatment was inconclusive as to whether the tracer specifically engages the 
CNS TrkB/C in vivo, however specific binding was indicated from blocking significant lung uptake of the tracer which may be linked to TrkB specific binding in pulmonary tissue [50]. An ${ }^{18} \mathrm{~F}$ derivative bearing an ${ }^{18} \mathrm{~F}$-fluoroethyl group at the 5-hydroxy moiety of a new GW441756 derivative, although being highly selective over other kinases and displaying generally favorable in vitro properties (low $\mathrm{IC}_{50}$ values in the nanomolar range for all Trk subtypes), showed unexpected susceptibility towards CYP450 metabolism in vitro in contrast to the original GW441756. These findings were corroborated by rapid formation of brain penetrating metabolites and no observable blocking upon pre-treatment with non-radioactive GW441756 in an animal PET experiment in rats. It can be concluded from our data that radioligands for pan-Trk imaging with PET, derived from the 3-indolydene 4-aza-2-oxindole scaffold despite being selective and highly potent inhibitors of Trk, do most probably not lend themselves towards PET tracer development and clinical translation. The main reason is the inevitable presence of the low affinity E-isomer of $\left[{ }^{11} \mathrm{C}\right] \mathrm{GW} 441756$ due to the rapid reaching of a photostationary state, a physicochemical reality that most definitely is not limited to GW441756 but rather extends to all structurally similar derivatives. However, both tracers displayed high specific binding in TrkB expressing neuroblastoma tumour sections in vitro providing a proof of principle of the utility of such radioligands for oncological imaging.

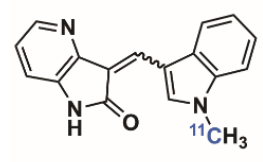

$\left[{ }^{11} \mathrm{C}\right] 1\left(\left[{ }^{11} \mathrm{C}\right] \mathrm{GW} 441756\right)$ 2015

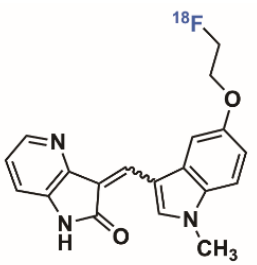

$\left[{ }^{18} \mathrm{~F}\right] 2$

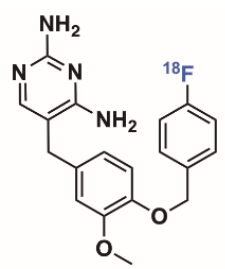

$\left[{ }^{18} \mathrm{~F}\right] 3\left(\left[{ }^{18} \mathrm{~F}\right]\right.$ FluoroGW2580) 2014

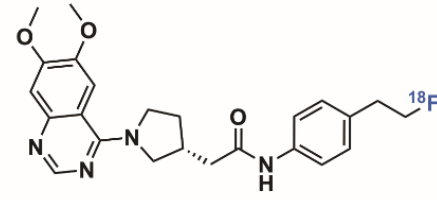

$\left[{ }^{18} \mathrm{~F}\right] 4\left(\left[{ }^{18} \mathrm{~F}\right] \mathrm{QMICF}\right)$ 2017

Figure 3. Chemical structures of early Trk-targeted positron emission tomography (PET) radioligands.

\subsubsection{2,4-Diaminopyridmidine and Quinazoline-Based Radioligands}

In order to explore the effect of targeting the DFG-out Trk kinase conformation, especially with regard to kinetics [51], we also developed type II radiolabeled inhibitors. In general, type II Trk inhibitors tend to possess mostly elongated structural features, higher molecular weight and HBD count than type-I inhibitors, which may be detrimental for BBB permeation but inconsequential in the context of peripheral imaging. So far we have developed two distinct type-II radiolabeled inhibitors belonging to the class of 2,4-diaminopyridines [52] and quinazoline [53] (Figure 3). Analyses of large kinase inhibitor sets identified GW2580, an orally active dual Trk/colony-stimulating factor-1 receptor (CSF-1R) inhibitor $\left(K_{\mathrm{d}} ; \mathrm{CSF}-1 \mathrm{R}=2 \mathrm{nM}\right.$, TrkA $=630 \mathrm{nM}$, $\operatorname{TrkB}=36 \mathrm{nM}$, and $\operatorname{TrkC}=120 \mathrm{nM}$ ), as one of the most kinome selective inhibitors known [54]. Based on this attribute, and the overall promising preclinical ADME (absorption, distribution, metabolism, and excretion) of this inhibitor, we undertook to identify a radioligand based on this scaffold. We rationalized that since CSF-1R is highly expressed in tumour-infiltrating macrophages, such compounds may be useful in cancer imaging beyond Trk. We synthesized fluorinated derivatives and performed labelling of the most promising lead, 5-(4-((4-[ $\left.{ }^{18} \mathrm{~F}\right]$ fluorobenzyl)oxy)-3-methoxybenzyl)pyrimidine-2,4-diamine $\left(\left[{ }^{18} \mathrm{~F}\right] 3\right.$, Figure 3$)$. While inhibitor $\left[{ }^{18} \mathrm{~F}\right] 3$ maintained the excellent kinome selectivity of GW2580, its radiosynthesis was challenging and relied on the synthesis of the prosthetic group $4-\left[{ }^{18} \mathrm{~F}\right]$ fluorobenzyl bromide [55] followed by subsequent reaction with a phenolic labeling precursor. The multistep procedure only produced low radiochemical yields (RCYs) of $\left[{ }^{18} \mathrm{~F}\right] 3$. This technical shortcoming precluded this tracer from its translation into a clinical setting and only most recent efforts applying Cu-mediated late stage fluorination techniques provided this tracer in high RCYs and high molar activities suitable for human PET scanning (unpublished). More recently, the quinazoline-based type II pan-Trk inhibitor $\left[{ }^{18} \mathrm{~F}\right] \mathrm{QMICF}\left(\left[{ }^{18} \mathrm{~F}\right] 4\right)$ was developed (Figure 3) [53]. Starting from the corresponding racemic quinazoline-based dual FLT3/TrkB inhibitor, a structure-activity relationship study demonstrated that the $(R)$-enantiomer was primarily responsible for Trk inhibition [56]. To conserve optimal target 
interaction with the Trk receptor, the position of fluorine introduction was carefully consolidated through molecular modeling. Only one particular position, namely the allosteric pocket fragment, proved adaptable towards structural modifications with the least detrimental effect on binding interaction. We replaced the isopropyl moiety connected in 4-position to a structurally flexible benzene moiety in the original inhibitor with a 2-fluoro ethyl group to facilitate an easy introduction of ${ }^{18} \mathrm{~F}^{-}$via common nucleophilic substitution of a corresponding mesyl derivative. This modification led to an about 10-fold potency reduction but also favorably abrogated FLT3 activity. Kinases profiling of inhibitor 4 revealed excellent selectivity and a Calcein-AM assay in P-gp overexpressing MDCKII cells confirmed only low interaction with P-gp. Radiotracer $\left[{ }^{18} \mathrm{~F}\right] \mathrm{QMICF}$ could be straightforwardly synthesized from the corresponding mesyl precursor in RCYs of $18 \%$ in one step. The compound is currently being evaluated for Trk brain imaging and tumor imaging in TrkA-TPM3 $\left(\mathrm{IC}_{50}=162 \mathrm{nM}\right)$ overexpressing colon carcinoma KM12 mice tumor xenografts and other neoplasms bearing diverse NTKR fusions.

\subsubsection{Imidazo[1,2-b]pyridazine-Based Radioligands}

In 2014, when we started the syntheses of imidazo[1,2-b]pyridazine-based radioligands, it was demonstrated that NTRK fusion were actionable drivers in human cancers, however only a few non selective Trk inhibitors had been used in a therapeutic approach. The Trk inhibitor landscape was however rapidly moving from exploratory tool inhibitor (such as GW441756) to more relevant drug leads and there was a clear trend in preclinical work and patent literature towards what has now become one of the most explored chemotypes, specifically imidazo[1,2-b]pyridazine and pyrazolo[1,5-a]pyrimidine-based inhibitors (Figure 4). Cognizant of this trend, and expecting that scaffolds selected for clinical oncological studies should display overall excellent druglike properties which could in turn be advantageous for achieving brain and peripheral imaging using PET, we reoriented our effort towards inhibitors of these classes. We found that in comparison to all potential radiotracers described above, the type I pan-Trk inhibitors sharing the (2-pyrrolidin-1-yl)imidazo[1,2-b]pyridazine structural motif (Figure 5), share exceptionally high affinities to all three Trk subtype receptors orders of magnitude higher than any other inhibitor described so far. ${ }^{57}$ Based on extensive molecular docking studies, a comprehensive PET-oriented library of imidazo[1,2-b]pyridazine-based compounds (bearing positions amenable for labeling using carbon-11 and/or fluorine-18) was reported in 2015 [57]. All new pan-Trk inhibitors were subjected to a $\left[\gamma^{-33} \mathrm{P}\right]$ ATP-based enzymatic assay to assess activities towards the different Trk subtypes. Remarkably, eight inhibitors from this library displayed $<200 \mathrm{pM}$ potency against TrkB/C and were initially regarded as potential candidates for radiotracer development. Two structures, $( \pm)$-IPMICF6 and $\left( \pm\right.$ )-IPMICF10 (Figure 5) lent themselves towards an easy introduction of ${ }^{18} \mathrm{~F}$ via simple nucleophilic substitution and were translated into the corresponding ${ }^{18} \mathrm{~F}$-radiotracers. Both compounds displayed favorable in vitro activity and kinome selectivity. Their ${ }^{18} \mathrm{~F}$ labeling was straightforward and yielded the corresponding radiotracers in 3\% and $8 \% \mathrm{RCY}$, respectively. In autoradiography experiments, both compounds were conclusively apt to topologically map the known brain distribution (cortex, striatum, thalamus and cerebellum) of TrkB/C receptors in coronal rat brain sections in accordance with mRNA and protein levels. In an important follow up study, one inhibitor from the reported imidazo[1,2-b]pyridazine-based library, IPMICF16, was chosen for radiotracer translation based on the alignment of favorable physicochemical properties (as described above) and optimal pharmacological parameters [58]. Pan-Trk inhibitor IPMICF16's structure is geared towards simple ${ }^{11} \mathrm{C}$-methylation radiolabeling. In order to confirm that $(R)$-IPMICF16 was the more active and hence more suitable enantiomer for radiotracer development, as expected based on crystal structure and docking analyses, both the $R$ and $S$ isomers were synthesized and evaluated. Non-radioactive $(R)$-IPMICF16 displayed $\mathrm{IC}_{50} \mathrm{~S}$ of 4.0, 0.2 and $0.1 \mathrm{nM}$ for human TrkA, TrkB, and TrkC, respectively and inhibitory constants $\left(K_{\mathrm{i}}\right)$ of 2.80, 0.05 and $0.021 \mathrm{nM}$ for TrkA, TrkB, and TrkC, respectively (200,000-500,000-fold over $K_{m}$ ATP for all Trks). The $S$-enantiomer was significantly less potent which is in line with our docking studies. Interestingly, this study showed that $(R)$-IPMICF16 constitutes a rare example where an ATP-competitive Trk inhibitor displays intra-Trk sub-type selectivity. With regard to TrkA, (R)-IPMICF16 showed 56-fold and 133-fold 
higher selectivity for TrkB and TrkC, respectively. It was further asserted that the compound shows high selectivity towards TrkB/C over $>99 \%$ of the testable human kinome (369 targets). The radiosynthesis of $\left[{ }^{11} \mathrm{C}\right]-(R)-$ IPMICF16 was routinely achieved using the corresponding desmethyl-precursor and radioactive $\left[{ }^{11} \mathrm{C}\right]$ methyl iodide (or alternatively $\left[{ }^{11} \mathrm{C}\right]$ methyl triflate) in $>10 \% \mathrm{RCYs}$. Soon thereafter, the robust synthesis was set up according to good manufacturing practices and regulations at four different production sites where $\left[{ }^{11} \mathrm{C}\right]-(R)-\mathrm{IPMICF} 16$ was evaluated in mice, rats, non-human primates, and finally in one healthy human subject (Figure 6).

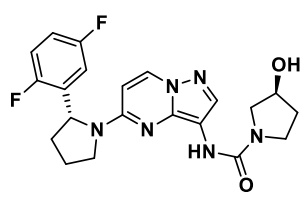

5 (Larotrectinib) Pyrazolo[1,5a]pyrimidines Multiple Phase I/II, 2015-

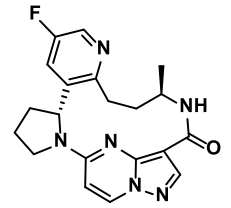

6 (LOXO-195)
Pyrazolo[1,5a]pyrimidines(macrocyclic)

Phase I/II, 2017-

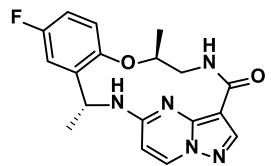

7 (Repotrectinib - TPX-0005)

(macrocyclic) Phase I/II, 2017-

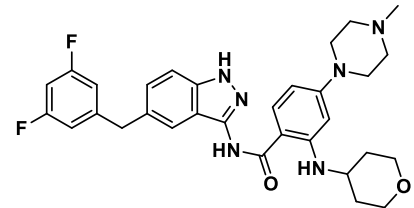

8 (Entrectinib)

Multiple Phase I/II, 2015-

Figure 4. Chemical structures of selected clinical Trk inhibitors.<smiles>NC(=O)c1cnc2ccc(N3CCC[C@H]3c3cccc(F)c3)nn12</smiles>

$\left[{ }^{11} \mathrm{C}\right]-( \pm)-$ IPMICF22 ([ $\left.\left.{ }^{11} \mathrm{C}\right] 9\right)$

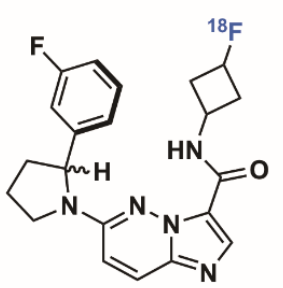

$\left[{ }^{18} \mathrm{~F}\right]-( \pm)-I P M I C F 6$ ( $\left.\left.{ }^{11} \mathrm{C}\right] 10\right)$

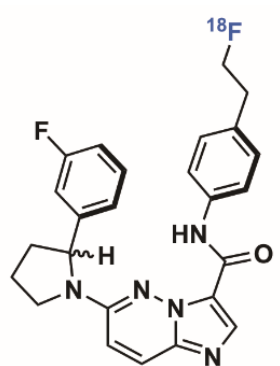

$\left[{ }^{18} \mathrm{~F}\right]-( \pm)-$ IPMICF10 $\left(\left[{ }^{11} \mathrm{C}\right] 11\right)$

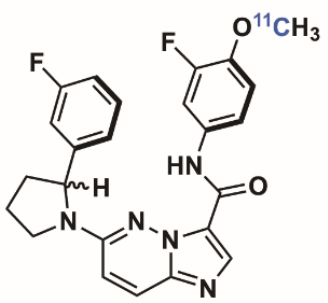

$\left[{ }^{11} \mathrm{C}\right]-( \pm)-$ IPMICF16 $\left(\left[{ }^{11} \mathrm{C}\right] 12\right)$

Figure 5. Chemical structures of preclinical imidazo[1,2b]pyridazine-based Trk-targeted PET radioligands.

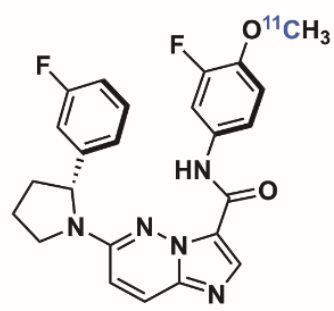

$\left[{ }^{11} \mathrm{C}\right]-(R)-I P M I C F 16$ $\left(\left[{ }^{11} \mathrm{C}\right]-(R)-12\right)$

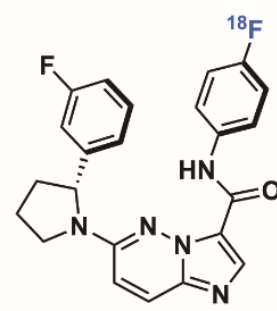

$\left[{ }^{18} \mathrm{~F}\right]$ TRACK

([ $\left.\left.{ }^{18} \mathrm{~F}\right]-(R)-13\right)$

Figure 6. Chemical structures of clinical imidazo[1,2b]pyridazine-based Trk-targeted PET radioligands.

Despite partial elimination of $\left[{ }^{11} \mathrm{C}\right]-(R)-$ IPMICF16 from the rat brain due to active efflux transporters in rodents (P-gp and breast cancer resistance protein) as demonstrated in double knockout $M d r 1 a / b-B c r p$ mice, SUV after 60-min scan time was still 0.4. The brain TrkB/C specific binding of $\left[{ }^{11} \mathrm{C}\right]-(R)-$-IPMICF16 in vivo was unambiguously demonstrated by pharmacological challenge with the clinical pan-Trk inhibitor entrectinib. Up to $88 \%$ of the radioactivity signal could be blocked after intraperitoneal injection of entrectinib, albeit high blocking doses $(350 \mathrm{mg} / \mathrm{kg})$ were required due to the documented low brain uptake of this inhibitor. Encouraged by these promising data, an evaluation of $\left[{ }^{11} \mathrm{C}\right]-(R)-\mathrm{IPMICF} 16$ was performed in non-human primates (NHP). In comparison to rodents, the brain kinetics in NHP was significantly slower with a continuously increasing radioactivity signal in the brain devoid of an observable washout throughout the entire 60 min PET scan. Regional analysis 
confirmed a heterogenous radioactivity distribution following Trk rich grey matter with highest uptake in thalamus (SUV 0.8). Radioactivity uptake in white matter was comparatively low, as expected and in accordance with low level Trk expression. Although the efflux kinetic profiles of $\left[{ }^{11} \mathrm{C}\right]-(R)-I P M I C F 16$ was notable in rodents, data from NHP PET imaging indicated interspecies differences and suggested that efflux may not be a liability in higher species. Hence, it was decided to move the tracer forward towards human in vivo translation. This decision was further motivated by the demonstration that in vitro [ $\left.{ }^{11} \mathrm{C}\right]-(R)-I P M I C F 16$ autoradiography was sufficiently sensitive to reproduce the known and significant region-specific hippocampal TrkB/C density reduction found in AD brains (compared to age-matched controls). All radioactivity signals in brain tissues (controls and AD) were blockable using a structurally unrelated pan-Trk inhibitor proving specificity of the observed uptake as a result of TrkB/C receptor engagement. Taken together, these preclinical data justified moving towards a first-in-human tracer evaluation with PET.

Injection of $\left[{ }^{11} \mathrm{C}\right]-(R)-I P M I C F 16$ into a 41-year-old healthy male subject led to brain uptake of the radiotracer which was rapid, with a peak SUV in the thalamus of 1.5 after $25 \mathrm{~s}$ post injection, very similar to what was observed in NHP. After reaching equilibrium, the SUV in one of the highest Trk tissues, the thalamus, remained 0.7 after 60 min with low observable washout. In accordance with non-human primate PET data, the radioactivity distribution in human matched the topography of the Trk disposition in grey matter with lowest SUVs in TrkB/C low white matter regions (Figure 7). The overall distribution aligned affirmatively with the known Trk distribution from ex vivo studies with highest uptake in the thalamus, followed by cerebellum and cortex. No adverse effects were observed after the injection of $\left[{ }^{11} \mathrm{C}\right]-(R)-I P M I C F 16$.

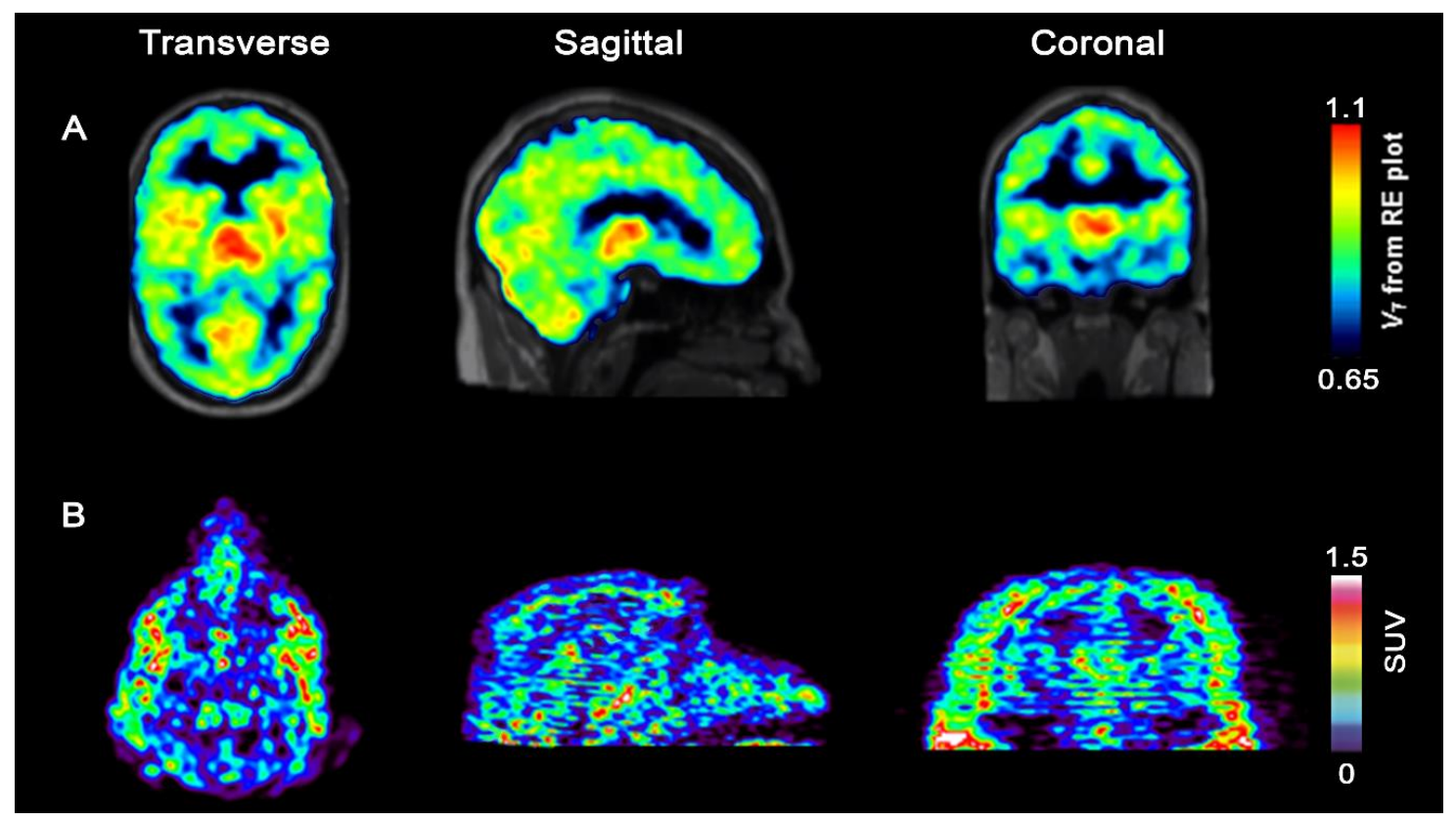

Figure 7. Upper row: PET/T1MP-RAGE MRT in vivo overlay images of $\left[{ }^{11} \mathrm{C}\right]-(\mathrm{R})-12$ in a human subject with high SUVs in TrkB/C rich compartments such as thalamus, followed by cerebellum and cortical grey matter and low uptake in Trk devoid white matter areas; Bottom row: In vivo PET images of $\left[{ }^{18} \mathrm{~F}\right]-(R)-13$ (high $A_{m}$ of $245 \mathrm{GBq} / \mu \mathrm{mol}$ ) in rhesus monkey brain matching the expected TrkB/C distribution.

Owing to the detailed structure-activity study conducted in the first phase of this project, we intended to modify the structure of $\left[{ }^{11} \mathrm{C}\right]-(R)-I P M I C F 16$ to enable labeling with fluorine-18 based on the recently introduced $\mathrm{Cu}$ catalysed late stage fluorination of non-activated aromatic boronic esters [59-61]. Despite several shortcomings such as the inevitable side formation of protodeboronation side products, consequently contaminating the ${ }^{18}$ F-labeled target molecule and therefore significantly 
reducing the effective molar activity $\left(A_{\mathrm{m}}\right)\left(\right.$ the ${ }^{18} \mathrm{~F}$ and $\mathrm{H}$ substitution product have very similar physicochemical as well as pharmacodynamics properties), the Cu-catalyzed ${ }^{18} \mathrm{~F}$ fluorination of boronic esters and the introduction of mesityl aryl iodonium salts as labeling precursors can be easily regarded as being among the most important developments of modern ${ }^{18} \mathrm{~F}$-radiochemistry $[62,63]$. $\left[{ }^{11} \mathrm{C}\right]-(R)-$ IPMICF16 originally bore a methoxy group directly adjacent to the fluorine atom at the aromatic amide fragment (Figure 6) which was identified as one contributor for the overall P-gp liability observed. That methoxy group was removed resulting in a pan-Trk inhibitor with further improved subtype selectivity but slightly reduced affinity for TrkB/C to reach binding equilibrium faster than $\left[{ }^{11} \mathrm{C}\right]-(R)-I P M I C F 16$. The efflux of the corresponding fluorinated inhibitor was also noticeably reduced in cells. This radiotracer, called $\left[{ }^{18} \mathrm{~F}\right]$ TRACK (the R isomer), was conveniently obtained in one step via $\mathrm{Cu}$ mediated radio-fluorination of a corresponding boronic ester precursor (the Bpin derivative) in satisfactory RCYs for further translation [64]. During the ${ }^{18} \mathrm{~F}$-fluorination, the protodeboronation product was formed which reduced the effective $A_{m}$ to a low value of $15 \mathrm{GBq} / \mu \mathrm{mol}$, an order of magnitude below what was expected. After extensive screening, this problem could be solved by employing a pentafluorophenyl (PFP) HPLC column for tracer purification-which constitutes currently the standard method used to achieve separation [65]. The column material showed improved stationary phase affinity interaction with the fluorinated TRACK tracer over standard octadecylsilane reversed phase, leading to a base-line separation of $\left[{ }^{18} \mathrm{~F}\right] \mathrm{TRACK}$ from all impurities-including the protodeboronation side product. This improved the $A_{\mathrm{m}}$ to $>100 \mathrm{GBq} / \mu \mathrm{mol}$. In vivo evaluation in rhesus monkey confirmed uptake of $\left[{ }^{18} \mathrm{~F}\right] \mathrm{TRACK}$ in TrkB/C-rich regions with SUVs in the grey matter ranging from 0.9 for the cerebellum, 0.8 for the thalamus, and 0.6 for the cortex (Figure 7). White matter uptake was comparably low (0.2 SUV). The brain kinetics of $\left[{ }^{18} \mathrm{~F}\right]$ TRACK were significantly different than what has been observed for $\left[{ }^{11} \mathrm{C}\right]-(R)$-IPMICF16, displaying characteristics of a more reversibly binding radiotracer. Injections of $\left[{ }^{18} \mathrm{~F}\right] \mathrm{TRACK}$ of varying $A_{\mathrm{m}}$ (low, medium and high) into the same monkey illustrated the paramount importance of high $A_{\mathrm{m}}$ for Trk PET imaging. The PET signal was obviously reduced in cases of sub-optimal $A_{\mathrm{m}} .\left[{ }^{18} \mathrm{~F}\right] \mathrm{TRACK}$ is currently evaluated in human healthy controls and first in human PET data will be reported in due course. First obtained results with $\left[{ }^{18} \mathrm{~F}\right]$ TRACK in a healthy human volunteer are in line with the data obtained with $\left[{ }^{11} \mathrm{C}\right]-(R)-$ IPMICF16 in terms of PET image quality and ease of production (unpublished).

\section{Conclusions}

The analysis, investigation and quantification of Trk receptors in neurodegenerative diseases and cancer is currently limited to ex vivo post-mortem analysis or invasive methods. While these kinases constitute important molecular targets which can be visualized using non-invasive PET imaging, Trk PET imaging is still in its infancy. Whether prototypical radioligands such as $\left[{ }^{11} \mathrm{C}\right]-(R)-$ IPMICF16 and $\left[{ }^{18} \mathrm{~F}\right]$ TRACK, which display moderate brain uptake in humans, will be suitable for clinical neuroimaging and can provide reliable CNS TrkB/C measurements or adequately detect reductions in receptor density remain to be established. While tracers with higher brain penetration, volume of distribution $\left(V_{\mathrm{T}}\right)$ values or binding potentials are desirable for temporally and spatially assessing Trk expression in conditions such as $\mathrm{AD}$, the current tracers could certainly be beneficial in cases where Trk is overexpressed such as in numerous human cancers, both in the periphery and CNS. Further research is currently aimed at identifying structural determinants improving overall brain uptake of current type I and II tracers, including cyclic derivatives such as LOXO 195 and derivatives thereof.

Funding: This research was funded by Cancer Research Society and C17 Council to R.S., Natural Science and Engineering Council of Canada (NSERC) to R.S., the Weston Brain Institute to R.S. and U.S Department of Energy/National Institute of Biomedical Imaging and Bioengineering (DE-SC0012484) to P.J.H.S.

Conflicts of Interest: The authors declare no conflict of interest. 


\section{References}

1. Lessmann, V.; Gottmann, K.; Malcangio, M. Neurotrophin secretion: Current facts and future prospects. Progr. Neurobiol. 2003, 69, 341-374. [CrossRef]

2. Chao, M.V. Neurotrophin receptors: A window into neuronal differentiation. Neuron 1992, 9, $583-593$. [CrossRef]

3. Chao, M.V. Neurotrophins and their receptors: A convergence point for many signalling pathways. Nat. Rev. Neurosci. 2003, 4, 299-309. [CrossRef] [PubMed]

4. Huang, E.J.; Reichardt, L.F. Trk Receptors: Roles in Neuronal Signal Transduction. Annu. Rev. Biochem. 2003, 72, 609-642. [CrossRef] [PubMed]

5. Kaplan, D.R.; Miller, F.D. Neurotrophin signal transduction in the nervous system. Curr. Opin. Neurobiol. 2000, 10, 381-391. [CrossRef]

6. Fenner, B.M. Truncated TrkB: Beyond a dominant negative receptor. Cytokine Growth Factor Rev. 2012, 23, 15-24. [CrossRef] [PubMed]

7. Yan, W.; Lakkaniga, N.R.; Carlomagno, F.; Santoro, M.; McDonald, N.Q.; Lv, F.; Gunaganti, N.; Frett, B.; Li, H.-Y. Insights into Current Tropomyosin Receptor Kinase (TRK) Inhibitors: Development and Clinical Application. J. Med. Chem. 2018. [CrossRef]

8. Huang, E.J.; Reichardt, L.F. Neurotrophins: Roles in neuronal development and function. Annu. Rev. Neurosci. 2001, 24, 677-736. [CrossRef]

9. Poo, M.-M. Neurotrophins as synaptic modulators. Nat. Rev. Neurosci. 2001, 2, 24-32. [CrossRef]

10. Zhang, F.; Kang, Z.; Li, W.; Xiao, Z.; Zhou, X. Roles of brain-derived neurotrophic factor/tropomyosin-related kinase B (BDNF/TrkB) signalling in Alzheimer's disease. J. Clin. Neurosci. 2012, 19, 946-949. [CrossRef]

11. Song, J.-H.; Yu, J.-T.; Tan, L. Brain-Derived Neurotrophic Factor in Alzheimer's Disease: Risk, Mechanisms, and Therapy. Mol. Neurobiol. 2015, 52, 1477-1493. [CrossRef] [PubMed]

12. Murer, M.G.; Yan, Q.; Raisman-Vozari, R. Brain-derived neurotrophic factor in the control human brain, and in Alzheimer's disease and Parkinson's disease. Progr. Neurobiol. 2001, 63, 71-124. [CrossRef]

13. Reinhart, V.; Bove, S.E.; Volfson, D.; Lewis, D.A.; Kleiman, R.J.; Lanz, T.A. Evaluation of TrkB and BDNF transcripts in prefrontal cortex, hippocampus, and striatum from subjects with schizophrenia, bipolar disorder, and major depressive disorder. Neurobiol. Dis. 2015, 77, 220-227. [CrossRef] [PubMed]

14. Oyesiku, N.M.; Evans, C.-O.; Houston, S.; Darrell, R.S.; Smith, J.S.; Fulop, Z.L.; Dixon, C.E.; Stein, D.G. Regional changes in the expression of neurotrophic factors and their receptors following acute traumatic brain injury in the adult rat brain. Brain Res. 1999, 833, 161-172. [CrossRef]

15. Deng, V.; Matagne, V.; Banine, F.; Frerking, M.; Ohliger, P.; Budden, S.; Pevsner, J.; Dissen, G.A.; Sherman, L.S.; Ojeda, S.R. FXYD1 is an MeCP2 target gene overexpressed in the brains of Rett syndrome patients and Mecp2-null mice. Hum. Mol. Genet. 2007, 16, 640-650. [CrossRef] [PubMed]

16. Gupta, V.K.; You, Y.; Gupta, V.B.; Klistorner, A.; Graham, S.L. TrkB Receptor Signalling: Implications in Neurodegenerative, Psychiatric and Proliferative Disorders. Int. J. Mol. Sci. 2013, 14, 10122-10142. [CrossRef]

17. Ferrer, I.; Marín, C.; Rey, M.J.; Ribalta, T.; Goutan, E.; Blanco, R.; Tolosa, E.; Martí, E. BDNF and Full-length and Truncated TrkB Expression in Alzheimer Disease. Implications in Therapeutic Strategies. J. Neuropathol. Exp. Neurol. 1999, 58, 729-739. [CrossRef]

18. Allen, S.J.; Wilcock, G.K.; Dawbarn, D. Profound and Selective Loss of Catalytic TrkB Immunoreactivity in Alzheimer's Disease. Biochem. Biophys. Res. Commun. 1999, 264, 648-651. [CrossRef]

19. Savaskan, E.; Müller-Spahn, F.; Olivieri, G.; Bruttel, S.; Otten, U.; Rosenberg, C.; Hulette, C.; Hock, C. Alterations in Trk A, Trk B and Trk C Receptor Immunoreactivities in Parietal Cortex and Cerebellum in Alzheimer's Disease. Eur. Neurol. 2000, 44, 172-180. [CrossRef]

20. Ginsberg, S.D.; Che, S.; Wuu, J.; Counts, S.E.; Mufson, E.J. Down regulation of trk but not p75NTR gene expression in single cholinergic basal forebrain neurons mark the progression of Alzheimer's disease. J. Neurochem. 2006, 97, 475-487. [CrossRef]

21. Castello, N.A.; Nguyen, M.H.; Tran, J.D.; Cheng, D.; Green, K.N.; LaFerla, F.M. 7,8-Dihydroxyflavone, a Small Molecule TrkB Agonist, Improves Spatial Memory and Increases Thin Spine Density in a Mouse Model of Alzheimer Disease-Like Neuronal Loss. PLoS ONE 2014, 9, e91453. [CrossRef] [PubMed]

22. Li, N.; Liu, G.-T. The novel squamosamide derivative FLZ enhances BDNF/TrkB/CREB signaling and inhibits neuronal apoptosis in APP/PS1 mice. Acta Pharmacol. Sin. 2010, 31, 265-272. [CrossRef] [PubMed] 
23. Massa, S.M.; Yang, T.; Xie, Y.; Shi, J.; Bilgen, M.; Joyce, J.N.; Nehama, D.; Rajadas, J.; Longo, F.M. Small molecule BDNF mimetics activate TrkB signaling and prevent neuronal degeneration in rodents. J. Clin. Investig. 2010, 120, 1774-1785. [CrossRef] [PubMed]

24. Nagahara, A.H.; Tuszynski, M.H. Potential therapeutic uses of BDNF in neurological and psychiatric disorders. Nat. Rev. Drug Discov. 2011, 10, 209-219. [CrossRef] [PubMed]

25. Devi, L.; Ohno, M. TrkB reduction exacerbates Alzheimer's disease-like signaling aberrations and memory deficits without affecting $\beta$-amyloidosis in 5XFAD mice. Transl. Psychiatry 2015, 5, e562. [CrossRef] [PubMed]

26. Géral, C.; Angelova, A.; Lesieur, S. From Molecular to Nanotechnology Strategies for Delivery of Neurotrophins: Emphasis on Brain-Derived Neurotrophic Factor (BDNF). Pharmaceutics 2013, 5, 127-167. [CrossRef] [PubMed]

27. Cocco, E.; Scaltriti, M.; Drilon, A. NTRK fusion-positive cancers and TRK inhibitor therapy. Nat. Rev. Clin. Oncol. 2018, 15, 731-747. [CrossRef] [PubMed]

28. Sun, X.; Xiao, Z.; Chen, G.; Han, Z.; Liu, Y.; Zhang, C.; Sun, Y.; Song, Y.; Wang, K.; Fang, F.; et al. A PET imaging approach for determining EGFR mutation status for improved lung cancer patient management. Sci. Transl. Med. 2018, 10. [CrossRef]

29. Tejeda, G.S.; Díaz-Guerra, M. Integral Characterization of Defective BDNF/TrkB Signalling in Neurological and Psychiatric Disorders Leads the Way to New Therapies. Int. J. Mol. Sci. 2017, 18, 268. [CrossRef]

30. Bernard-Gauthier, V.; Boudjemeline, M.; Rosa-Neto, P.; Thiel, A.; Schirrmacher, R. Towards tropomyosinrelated kinase B (TrkB) receptor ligands for brain imaging with PET: Radiosynthesis and evaluation of 2-(4- $\left[{ }^{18} \mathrm{~F}\right]$ fluorophenyl)-7,8-dihydroxy-4H-chromen-4-one and 2-(4-([N-methyl-11C]-dimethylamino)phenyl)7,8-dihydroxy-4H-chromen-4-one. Bioorganic Med. Chem. 2013, 21, 7816-7829. [CrossRef]

31. Jang, S.-W.; Liu, X.; Yepes, M.; Shepherd, K.R.; Miller, G.W.; Liu, Y.; Wilson, W.D.; Xiao, G.; Blanchi, B.; Sun, Y.E.; et al. A selective TrkB agonist with potent neurotrophic activities by 7,8-dihydroxyflavone. Proc. Natl. Acad. Sci. USA 2010, 107, 2687-2692. [CrossRef]

32. Liu, X.; Chan, C.-B.; Jang, S.-W.; Pradoldej, S.; Huang, J.; He, K.; Phun, L.H.; France, S.; Xiao, G.; Jia, Y.; et al. A Synthetic 7,8-Dihydroxyflavone Derivative Promotes Neurogenesis and Exhibits Potent Antidepressant Effect. J. Med. Chem. 2010, 53, 8274-8286. [CrossRef]

33. Liu, X.; Chan, C.-B.; Qi, Q.; Xiao, G.; Luo, H.R.; He, X.; Ye, K. Optimization of a Small Tropomyosin-Related Kinase B (TrkB) Agonist 7,8-Dihydroxyflavone Active in Mouse Models of Depression. J. Med. Chem. 2012, 55, 8524-8537. [CrossRef] [PubMed]

34. Boltaev, U.; Meyer, Y.; Tolibzoda, F.; Jacques, T.; Gassaway, M.; Xu, Q.; Wagner, F.; Zhang, Y.-L.; Palmer, M.; Holson, E.; et al. Multiplex quantitative assays indicate a need for reevaluating reported small-molecule TrkB agonists. Sci. Signal. 2017, 10. [CrossRef] [PubMed]

35. Shia, C.-S.; Tsai, S.-Y.; Kuo, S.-C.; Hou, Y.-C.; Chao, P.-D.L. Metabolism and Pharmacokinetics of 3,3', $4^{\prime}, 7$-Tetrahydroxyflavone (Fisetin), 5-Hydroxyflavone, and 7-Hydroxyflavone and Antihemolysis Effects of Fisetin and Its Serum Metabolites. J. Agric. Food Chem. 2009, 57, 83-89. [CrossRef] [PubMed]

36. Jäger, A.; Saaby, L. Flavonoids and the CNS. Molecules 2011, 16, 1471-1485. [CrossRef]

37. Aldrich, C.; Bertozzi, C.; Georg, G.I.; Kiessling, L.; Lindsley, C.; Liotta, D.; Merz, K.M., Jr.; Schepartz, A.; Wang, S. The Ecstasy and Agony of Assay Interference Compounds. ACS Cent. Sci. 2017, 3, 143-147. [CrossRef]

38. Bothwell, M. Recent advances in understanding neurotrophin signaling. F1000Research 2016, 5. [CrossRef]

39. Altar, C.A.; Burton, L.E.; Bennett, G.L.; Dugich-Djordjevic, M. Recombinant human nerve growth factor is biologically active and labels novel high-affinity binding sites in rat brain. Proc. Nat. Acad. Sci. USA 1991, 88, 281-285. [CrossRef]

40. Altar, C.; Dugich-Djordjevic, M.; Armanini, M.; Bakhit, C. Medial-to-lateral gradient of neostriatal NGF receptors: Relationship to cholinergic neurons and NGF-like immunoreactivity. J. Neurosci. 1991, 11, 828-836. [CrossRef]

41. Merlio, J.P.; Ernfors, P.; Jaber, M.; Persson, H. Molecular cloning of rat trkC and distribution of cells expressing messenger RNAs for members of the trk family in the rat central nervous system. Neuroscience 1992, 51, 513-532. [CrossRef] 
42. Anderson, K.D.; Alderson, R.F.; Altar, C.A.; DiStefano, P.S.; Corcoran, T.L.; Lindsay, R.M.; Wiegand, S.J. Differential distribution of exogenous BDNF, NGF, and NT-3 in the brain corresponds to the relative abundance and distribution of high-affinity and low-affinity neurotrophin receptors. J. Comp. Neurol. 1995, 357, 296-317. [CrossRef] [PubMed]

43. Altar, C.A.; Siuciak, J.A.; Wright, P.; Ip, N.Y.; Lindsay, R.M.; Wiegand, S.J. In Situ Hybridization of trkB and trkC Receptor mRNA in Rat Forebrain and Association with High-affinity Binding of [ $\left.{ }^{125} \mathrm{I}\right] \mathrm{BDNF}$, $\left[{ }^{125} \mathrm{I}\right] \mathrm{NT}-4 / 5$ and $\left[{ }^{125} \mathrm{I}\right] \mathrm{NT}-3$. Eur. J. Neurosci. 1994, 6, 1389-1405. [CrossRef]

44. Zhang, L.; Villalobos, A.; Beck, E.M.; Bocan, T.; Chappie, T.A.; Chen, L.; Grimwood, S.; Heck, S.D.; Helal, C.J.; Hou, X.; et al. Design and Selection Parameters to Accelerate the Discovery of Novel Central Nervous System Positron Emission Tomography (PET) Ligands and Their Application in the Development of a Novel Phosphodiesterase 2A PET Ligand. J. Med. Chem. 2013, 56, 4568-4579. [CrossRef] [PubMed]

45. Pike, V.W. PET radiotracers: Crossing the blood-brain barrier and surviving metabolism. Trends Pharmacol. Sci. 2009, 30, 431-440. [CrossRef] [PubMed]

46. Rankovic, Z. CNS Drug Design: Balancing Physicochemical Properties for Optimal Brain Exposure. J. Med. Chem. 2015, 58, 2584-2608. [CrossRef] [PubMed]

47. Wager, T.T.; Hou, X.; Verhoest, P.R.; Villalobos, A. Moving beyond Rules: The Development of a Central Nervous System Multiparameter Optimization (CNS MPO) Approach to Enable Alignment of Druglike Properties. ACS Chem. Neurosci. 2010, 1, 435-449. [CrossRef] [PubMed]

48. Wood, E.R.; Kuyper, L.; Petrov, K.G.; Hunter, R.N.; Harris, P.A.; Lackey, K. Discovery and in vitro evaluation of potent TrkA kinase inhibitors: Oxindole and aza-oxindoles. Bioorg. Med. Chem. Lett. 2004, 14, 953-957. [CrossRef]

49. Bernard-Gauthier, V.; Aliaga, A.; Aliaga, A.; Boudjemeline, M.; Hopewell, R.; Kostikov, A.; Rosa-Neto, P.; Thiel, A.; Schirrmacher, R. Syntheses and Evaluation of Carbon-11- and Fluorine-18-Radiolabeled pan-Tropomyosin Receptor Kinase (Trk) Inhibitors: Exploration of the 4-Aza-2-oxindole Scaffold as Trk PET Imaging Agents. ACS Chem. Neurosci. 2015, 6, 260-276. [CrossRef]

50. Prakash, Y.; Thompson, M.A.; Meuchel, L.; Pabelick, C.M.; Mantilla, C.B.; Zaidi, S.; Martin, R.J. Neurotrophins in lung health and disease. Expert Rev. Respir. Med. 2010, 4, 395-411. [CrossRef]

51. Stachel, S.J.; Sanders, J.M.; Henze, D.A.; Rudd, M.T.; Su, H.-P.; Li, Y.; Nanda, K.K.; Egbertson, M.S.; Manley, P.J.; Jones, K.L.G.; et al. Maximizing Diversity from a Kinase Screen: Identification of Novel and Selective pan-Trk Inhibitors for Chronic Pain. J. Med. Chem. 2014, 57, 5800-5816. [CrossRef] [PubMed]

52. Bernard-Gauthier, V.; Schirrmacher, R. 5-(4-((4-[ $\left.{ }^{18} \mathrm{~F}\right]$ fluorobenzyl)oxy)-3-methoxybenzyl)pyrimidine-2,4diamine: A selective dual inhibitor for potential PET imaging of Trk/CSF-1R. Bioorg. Med. Chem. Lett. 2014, 24, 4784-4790. [CrossRef] [PubMed]

53. Bernard-Gauthier, V.; Mahringer, A.; Vesnaver, M.; Fricker, G.; Schirrmacher, R. Design and synthesis of a fluorinated quinazoline-based type-II Trk inhibitor as a scaffold for PET radiotracer development. Bioorg. Med. Chem. Lett. 2017, 27, 2771-2775. [CrossRef] [PubMed]

54. Davis, M.I.; Hunt, J.P.; Herrgard, S.; Ciceri, P.; Wodicka, L.M.; Pallares, G.; Hocker, M.; Treiber, D.K.; Zarrinkar, P.P. Comprehensive analysis of kinase inhibitor selectivity. Nat. Biotechnol. 2011, 29, 1046-1051. [CrossRef] [PubMed]

55. Lemaire, C.; Libert, L.; Plenevaux, A.; Aerts, J.; Franci, X.; Luxen, A. Fast and reliable method for the preparation of ortho- and para- $\left[{ }^{18} \mathrm{~F}\right]$ fluorobenzyl halide derivatives: Key intermediates for the preparation of no-carrier-added PET aromatic radiopharmaceuticals. J. Fluor. Chem. 2012, 138, 48-55. [CrossRef]

56. Baindur, N.; Gaul, M.D.; Kreutter, K.D.; Baumann, C.A.; Kim, A.J.; Xu, G.; Tuman, R.W.; Johnson, D.L. Alkylquinoline and Alkylquinazoline Kinase Modulators. U.S. Patent US2006281772, 14 December 2006.

57. Bernard-Gauthier, V.; Bailey, J.J.; Aliaga, A.; Kostikov, A.; Rosa-Neto, P.; Wuest, M.; Brodeur, G.M.; Bedell, B.J.; Wuest, F.; Schirrmacher, R. Development of subnanomolar radiofluorinated (2-pyrrolidin-1-yl)imidazo [1,2-b]pyridazine pan-Trk inhibitors as candidate PET imaging probes. MedChemComm 2015, 6, 2184-2193. [CrossRef]

58. Bernard-Gauthier, V.; Bailey, J.J.; Mossine, A.V.; Lindner, S.; Vomacka, L.; Aliaga, A.; Shao, X.; Quesada, C.A.; Sherman, P.; Mahringer, A.; et al. A Kinome-Wide Selective Radiolabeled TrkB/C Inhibitor for in Vitro and in Vivo Neuroimaging: Synthesis, Preclinical Evaluation, and First-in-Human. J. Med. Chem. 2017, 60, 6897-6910. [CrossRef] 
59. Preshlock, S.; Tredwell, M.; Gouverneur, V. ${ }^{18}$ F-Labeling of Arenes and Heteroarenes for Applications in Positron Emission Tomography. Chem. Rev. 2016, 116, 719-766. [CrossRef]

60. Brooks, A.F.; Topczewski, J.J.; Ichiishi, N.; Sanford, M.S.; Scott, P.J.H. Late-stage [(18)F]Fluorination: New Solutions to Old Problems. Chem. Sci. 2014, 5, 4545-4553.

61. Zlatopolskiy, B.D.; Zischler, J.; Krapf, P.; Zarrad, F.; Urusova, E.A.; Kordys, E.; Endepols, H.; Neumaier, B. Copper-Mediated Aromatic Radiofluorination Revisited: Efficient Production of PET Tracers on a Preparative Scale. Chem. A Eur. J. 2015, 21, 5972-5979. [CrossRef] [PubMed]

62. Tredwell, M.; Preshlock, S.M.; Taylor, N.J.; Gruber, S.; Huiban, M.; Passchier, J.; Mercier, J.; Génicot, C.; Gouverneur, V. A General Copper-Mediated Nucleophilic ${ }^{18}$ F Fluorination of Arenes. Angew. Chem. Int. Ed. 2014, 53, 7751-7755. [CrossRef] [PubMed]

63. Ichiishi, N.; Brooks, A.F.; Topczewski, J.J.; Rodnick, M.E.; Sanford, M.S.; Scott, P.J.H. Copper-catalyzed $\left[{ }^{18} \mathrm{~F}\right]$ fluorination of (mesityl)(aryl)iodonium salts. Org. Lett. 2014, 16, 3224-3227. [CrossRef] [PubMed]

64. Bernard-Gauthier, V.; Mossine, A.V.; Mahringer, A.; Aliaga, A.; Bailey, J.J.; Shao, X.; Stauff, J.; Arteaga, J.; Sherman, P.; Grand'Maison, M.; et al. Identification of [ ${ }^{18}$ F]TRACK, a Fluorine-18-Labeled Tropomyosin Receptor Kinase (Trk) Inhibitor for PET Imaging. J. Med. Chem. 2018, 61, 1737-1743. [CrossRef] [PubMed]

65. Mossine, A.V.; Brooks, A.F.; Bernard-Gauthier, V.; Bailey, J.J.; Ichiishi, N.; Schirrmacher, R.; Sanford, M.S.; Scott, P.J.H. Automated Synthesis of PET Radiotracers by Copper-mediated ${ }^{18}$ F-Fluorination of Organoborons: Importance of the Order of Addition and Competing Protodeborylation. J. Label. Compd. Radiopharm. 2018, 63, 228-236. [CrossRef] [PubMed]

(C) 2019 by the authors. Licensee MDPI, Basel, Switzerland. This article is an open access article distributed under the terms and conditions of the Creative Commons Attribution (CC BY) license (http:/ / creativecommons.org/licenses/by/4.0/). 